\title{
Promotion of adventitious root formation in root-cut mung bean seedlings by a commercial alkaline hydrolysis product of soy oil and its ingredients, unsaturated long-chain fatty acids
}

\author{
Shigeru Satoh, Taira Okubo and Yoshihiro Nomura \\ Faculty of Agriculture, Ryukoku University, Otsu 520-2194, Japan \\ Corresponding author: S. Satoh, E-mail: ssatoh@agr.ryukoku.ac.jp, Phone: +81-77-599-5700
}

Received on June 17, 2019; Accepted on July 31, 2019

\begin{abstract}
Synthetic auxins are currently used to promote adventitious root formation and thereby propagation by cutttage of plants in horticulture. In the present study, we tested commercial products, named NSC-Base ${ }^{\circledR}$ and NSC-Pro ${ }^{\circledR}$, which are alkaline hydrolysis products of soy oil and pine seed oil, respectively, for possible use as agents to promote adventitious root formation. The commercial products were found to stimulate adventitious root formation in cut stems of ornamental cabbage, and in root-cut seedlings of mung bean, cucumber, sweet pepper, tomato, melon and pumpkin. Their root-forming activity was further characterized using NSC-Base ${ }^{\circledR}$ and mung bean seedlings. NSCBase $^{\circledR}$ is mainly composed of sodium salts of long chain fatty acids. NSC-Base ${ }^{\circledR}$ diluted to $10^{4}$ to $10^{2}$ times and sodium linoleate, the major ingredient of NSC-B ${ }^{\circledR}$, at 0.1 and $1.0 \mathrm{mM}$, significantly increased adventitious root formation, when judged by the total root length and number of roots. Among sodium salts of long chain fatty acids included in NSCBase $^{\circledR}$, sodium salts of unsaturated fatty acids, linoleic acid, linolenic acid and probably oleic acid at $1 \mathrm{mM}$, were active in promoting adventitious root formation, but those of saturated fatty acids were not. Simultaneous application of NSC-Base ${ }^{\circledR}$ with 3-pyridinecarboxylic acid, a stimulator of root elongation, exerted a synergistic effect on root elongation in root-cut mung bean seedlings.
\end{abstract}

Keywords: adventitious root formation, long-chain fatty acids, sodium linoleate, soybean oil saponificate, Vigna radiata

Abbreviations: NSC-B ${ }^{\circledR}$, Nano Soy Colloid-Base ${ }^{\circledR}$;
IAA, indole-3-acetic acid; IBA, indole-3-butyric acid; 3-PCA, 3-pyridinecarboxylic acid

\section{Introduction}

Root growth is sensitive to exogenously applied auxin. Exogenous IAA promotes root elongation at very low concentrations $\left(10^{-8} \mathrm{M}\right.$ or less), but inhibits it significantly at higher concentrations $\left(10^{-4}\right.$ to $10^{-6}$ M) (Wightman et al. 1980, Knee and Hangarter 1996, Hopkins 1995, Sirivastava 2001). Moreover, IAA has been shown to stimulate root initiation from stem cuttings, thereby substantiating a practical use for auxins. In horticulture, the stimulatory effect of auxin on the formation of adventitious roots has been useful for the vegetative propagation of plants by cutting (Bernasconi 1998). However, auxin-type root forming agents sometimes inhibit the growth of initiated roots at the auxin concentrations used for root initiation (Arteca 1996).

Currently, synthetic auxins like IBA or 1 -naphtyleneacetamide are used to promote adventitious root formation and thereby propagation by cuttage of plants in horticulture. These synthetic auxins are used instead of the naturally occurring IAA because they are less metabolized by IAA oxidase or other enzymes and will persist in the tissue for longer periods of time (Arteca 1996). However, their usage is limited to some fruit and flowering woody plants because of the limited plant species that respond to exogenous auxins and safety concern to human health when used on some food vegetables. New chemicals that can be used on a wide range of plants and that are safe to human health need to be developed.

Satoh S, Okubo T, Nomura Y 2019 Promotion of adventitious root formation in root-cut mung bean seedlings by a commercial alkaline hydrolysis product of soy oil and its ingredients, unsaturated long-chain fatty acids. Plant Root 13:15-22. doi:10.3117/plantroot.13.15 
Recently, we tested commercial products, named NSC-Base ${ }^{\circledR}$ and NSC-Pro ${ }^{\circledR}$, which are alkaline hydrolysis products of soy oil and pine seed oil, respectively, as possible flower care agents to prolong vase life of cut ornamental flowers. Unexpectedly, however, we found that both agents could stimulate adventitious root formation in rootcut seedlings and stems of some vegetables. In the present study, we characterized the action of NSCBase $^{\circledR}$, using several plant species and active ingredients of NSC-Base ${ }^{\circledR}$, and their interaction with a root elongation stimulator, 3-pyridinecarboxylic acid (Satoh and Nomura 2017).

\section{Materials and Methods}

\section{Plant materials}

Seeds of ornamental cabbage (Brassica oleracea var. acephala f. tricolor

'Hatsubeni F1'), cucumber (Cucumis sativus 'Aonagakei-jibae'), and sweet pepper (Capasicum annuиm 'Fusimi-amanaga'), were purchased from Takii \& Co., Ltd, Kyoto, Japan, and those of pumpkin (Cucurbita sp. 'Stark') and melon (Cucumis melo 'Double Guard Power') were the gifts from the company. Mung bean (Vigna radiata) seeds were purchased from Nakahara Seed Product Co., Ltd., Fukuoka, Japan.

Qualitative observation for promotion of adventitious root formation in various plants

Ornamental cabbage: Ornamental cabbage seeds were sown in plastic containers (Relief Planter RP650-TB, $65.3(\mathrm{~W}) \times 24.5(\mathrm{D}) \times 18.5(\mathrm{H}) \mathrm{cm}$, net soil volume $16 \mathrm{~L}$, Iris Ohyama Inc. Sendai, Japan) filled with an organic growing medium (Hana-to-yasaino-tsuchi, Takii \& Co., Ltd) on September 23, 2017. Germinated seedlings were cultivated in an unheated greenhouse until harvest on February 18, 2018. Shoots with 15 to $20 \mathrm{~cm}$-long stems were cut out from the plants with a razor blade. These shoots were placed in a $0.9 \mathrm{~L}$ glass jar with stem ends in 0.4 $\mathrm{L}$ of respective test solutions. The test solutions were water (control) and 100-, 500- and $1 \times$ $10^{3}$-times dilutes (diluted with water) of NSC-Pro ${ }^{\circledR}$, an alkaline hydrolysis product of pine seed oil. The plants were left for 3 weeks at $23^{\circ} \mathrm{C}$ under continuous room light from white fluorescent lamps $\left(>15 \mu \mathrm{mol} \mathrm{m} \mathrm{m}^{-2}\right.$ PPFD) at the plant height level. The test solution in the glass jars was replenished every week. Experiments were conducted with three replicates. Profiles for adventitious root formation on the stem ends were observed with the naked eye and photographed with two replicates showing typical and almost identical responses.

Mung bean, cucumber, sweet pepper: Experiments were conducted in the spring (April-May) of 2018. Seeds were sown in Jiffy Pots Strips (\#517, BFG Supply Co., Burton, OH, USA), two seeds per cell, filled with the above described growing media. Seeds were geminated and seedlings were grown in an unheated greenhouse at natural day-night temperature fluctuation under natural day-night light cycle. Mung bean seeds were sown on April 18 and grown to April 30 (plant height ca. $10 \mathrm{~cm}$ ). Stems with two true leaves were cut two $\mathrm{cm}$ below the trace of fallen cotyledons. The seedlings were placed in a $5.5-\mathrm{ml}$ plastic tube with their cut stem end in $4.5 \mathrm{ml}$ test solutions, and left for one week at $23-25^{\circ} \mathrm{C}$ under continuous room light described above. The test solutions were water (control) and NSC-Base ${ }^{\circledR}$ (an alkaline hydrolysis product of soy oil) diluted $2 \times 10^{3}$ times with water. The test solutions were replenished once three or four days after the start of the experiment. After one week, the treated seedlings were observed for adventitious root formation and photographed. With cucumber, seeds were sown on April 19, 2018 and grown until May 1, 2018. Seedlings with 4-cm long hypocotyls below cotyledons were cut out, and treated in the same way as mung bean seedlings. Sweet pepper seeds were sown on April 19, 2018 and grown until May 19, when the seedlings had two cotyledons and four true leaves. Seedlings were cut two $\mathrm{cm}$ below the cotyledons and the apical half of each leaves was trimmed. The root-cut sweet pepper seedlings were treated as described above but left for ten days until May 29. Experiments were conducted with three replicates. Profiles for adventitious root formation on the stem ends were observed with the naked eye and photographed with two replicates showing typical and almost identical responses.

Qualitative analysis for promotion of adventitious root formation in root-cut mung bean seedlings

Experiments were conducted in the spring of 2019, from late March to the beginning of May. Procedures were followed to those described above for mung bean seedlings, but the cultivation of mung bean seedlings and treatment of root-cut seedlings were conducted under precisely controlled conditions; incubation at $26^{\circ} \mathrm{C}$ and under the $16-\mathrm{h}$ light (135 $\mu \mathrm{mol} \mathrm{m}^{-2} \mathrm{~s}^{-1}$ PPFD) and 8-h dark regime, and 6-day cultivation for seedlings growth (attained plant height of 6-7 cm) and 5-day incubation for adventitious root formation. Root-cut seedlings were prepared as described above. They were treated with NSC-Base ${ }^{\circledR}$ diluted to $10^{2}, 10^{3}, 10^{4}$ and $10^{5}$ times 
with water, and the control was water. In separate experiments, root-cut seedlings were treated with sodium linoleate at 0 (water control), $0.001,0.01$, 0.1 and $1.0 \mathrm{mM}$. The stock solution of sodium linoleate was first prepared at $1 \mathrm{mM}$ by moderate heating the solution until it became transparent. But after cooling the solution down to room temperature, it became turbid making a mixture of probably free acids, colloidal particles and precipitates of sodium linoleate, which surely reduced the actual concentration of free acids to below $1 \mathrm{mM}$. Irrespective of this defect, we used the solution (mixture) by referring its concentration as $1 \mathrm{mM}$, the initial concentration of added sodium linoleate. The sodium linoleate solutions less than $0.1 \mathrm{mM}$ were made by diluting the stock solution with water. We handled similarly the solutions of sodium salts of fatty acids other than sodium linoleate used later (shown in Fig. 4). The number of adventitious roots formed was counted by observing with naked eyes under a magnifier. Then, roots longer than $0.5 \mathrm{~mm}$ were detached from each seedling using a razor blade and photographed. The root length of each seedling was measured with a curvemeter (Pen-type Map-meter Concurve 10, Koizumi Sokki Mfg. Co., Ltd. Nagaoka, Japan) on printed photographs (Satoh and Nomura, 2017).

The effect of sodium salts of fatty acids in NSC-Base ${ }^{\circledR}$, was examined by incubating the rootcut mung bean seedlings in each of the fatty acids at $1 \mathrm{mM}$ as described above. The sodium salts of fatty acids were those of palmitic acid (C16:0), stearic acid (C18:0), oleic acid (C18:1), linoleic acid (C18:2) and linolenic acid (C18:3). Furthermore, to see a synergistic effect of NSC-Base ${ }^{\circledR}$ and 3-PCA, a previously-shown stimulator of root elongation (Satoh and Nomura, 2017), root-cut mung bean seedlings were treated alone or in combination with 3000 -fold diluted NSC-Base ${ }^{\circledR}$ or $0.3 \mathrm{mM} 3$-PCA. Number of roots formed and total length of roots per each seedling were measured as described above. The control was treated with water. All experiments described above were conducted with five replicated seedlings, and repeated four times.

\section{Statistical analysis}

Statistical analyses were carried out by Dunnett's multiple range test using an online statistical analysis program, MEPHAS (http://www.gen-info. osaka-u.ac.jp/testdocs/tomocom/).

\section{Chemicals}

NSC-Base ${ }^{\circledR}$ and NSC-Pro ${ }^{\circledR}$ were gifts from WIT Co., Ltd, Hirakata, Osaka, Japan. Sodium salt of linoleic acid was purchased first from Sigma-Aldrich. Co., St. Louis, Mo, USA, and later from Tokyo Chemical Industry, Co. Ltd, Tokyo Japan. Other specimens of long-chain fatty acid sodium salts were purchased from Tokyo Chemical Industry, Co. Ltd, Tokyo Japan, but linolenic acid from Wako Pure Chemical Industries, Ltd., Osaka, Japan.

\section{Results and Discussion}

\section{Promotion by NSC-Pro ${ }^{\circledR}$ or NSC-Base ${ }^{\circledR}$ of adventitious root formation in various plants}

Figure 1 shows that NSC-Pro ${ }^{\circledR}$ or NSC-Base ${ }^{\circledR}$ promoted adventitious root formation in cut stems of ornamental cabbage shoots and cut seedlings of mung bean, cucumber and sweet pepper. Treatment with NSC-Pro ${ }^{\circledR}$ diluted 500 and $1 \times 10^{3}$ times markedly stimulated rooting of ornamental cabbage (cultivar 'Hatsubeni F1') stems, but that diluted 100 times caused no rooting. In the latter case, rooting was probably inhibited by the deleterious side effect at the high concentration. The adventitious root formation was also promoted by NSC-Pro ${ }^{\circledR}$ diluted 2 $\times 10^{3}$ times in cut shoots of another cultivar 'Haresugata F1' of ornamental cabbage (data not shown).

After the above described experiments, we suspected that the promotion of adventitious root formation might be caused by the action of sodium fatty acids included in NSC-Pro ${ }^{\circledR}$, which was substantiated later. NSC-Pro ${ }^{\circledR}$ is produced by alkaline hydrolysis of pine seed oil, which consists of triglycerides whose fatty acid composition is similar to that of soy oil (shown below), excepting the pine seed oil contains pinolenic acid instead of linolenic acid in soy oil. Pinolenic acid is an unusual fatty acid, which is an isomer of $\gamma$-linolenic acid. The prospective practical use of NSC-Pro ${ }^{\circledR}$ may provoke health or environmental concerns when used in the cultivation of vegetable seedlings. Therefore, in the following experiments, we used NSC-Base ${ }^{\circledR}$, which contains linolenic acid instead of pinolenic acid.

Treatment with NSC-Base ${ }^{\circledR}$ diluted $2 \times 10^{3}$ times promoted rooting in root-cut seedlings of mung bean, cucumber and sweet pepper. Also, NSC-Base ${ }^{\circledR}$ treatment promoted the formation of adventitious root in root-cut seedlings of cabbage, eggplant, melon, pumpkin and tomato seedlings (data not shown). The promotion of adventitious root formation was caused by NSC-Base ${ }^{\circledR}$ diluted 1 $\times 10^{3}$ to $2 \times 10^{3}$ times. These findings together suggest that NSC-Base ${ }^{\circledR}$ can promote adventitious root formation in Brassicaceae, Solanaceae, Cucurbitaceae, and Fabaceae plants. 

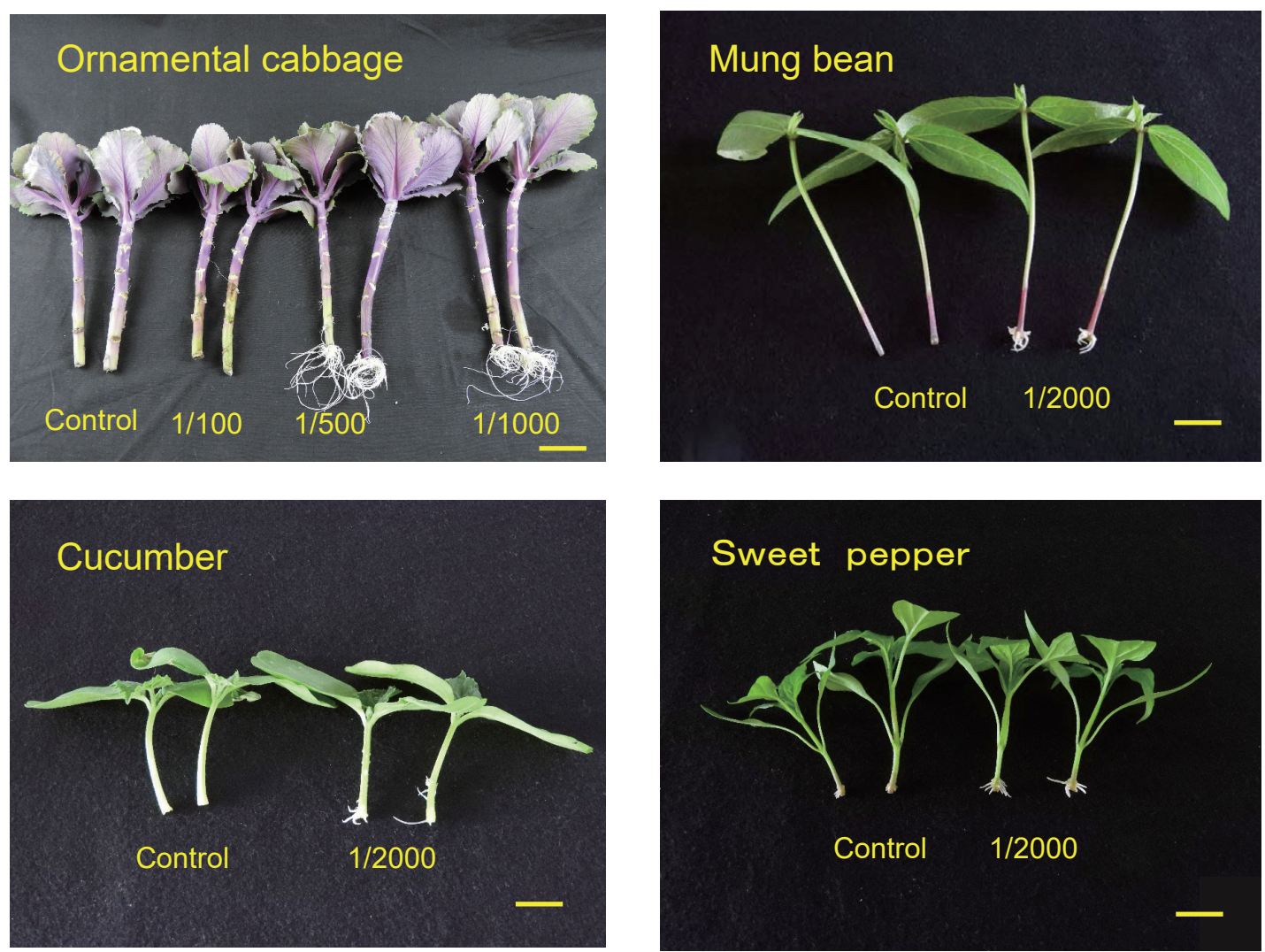

Fig. 1. Stimulation of adventitious root formation in root-cut ornamental cabbage stems, and mung bean, cucumber and sweet pepper seedlings by treatment with NSC-Pro ${ }^{\circledR}$ (ornamental cabbage) and NSC-Base ${ }^{\circledR}$ (other three plants). The concentrations of $\mathrm{NSC}^{\circledR}$ are shown by the magnitude of dilution of commercial NSC ${ }^{\circledR}$ products. Scale bars indicate $2 \mathrm{~cm}$ for mung bean, cucumber and sweet pepper seedlings, but $3 \mathrm{~cm}$ for ornamental cabbage plants.
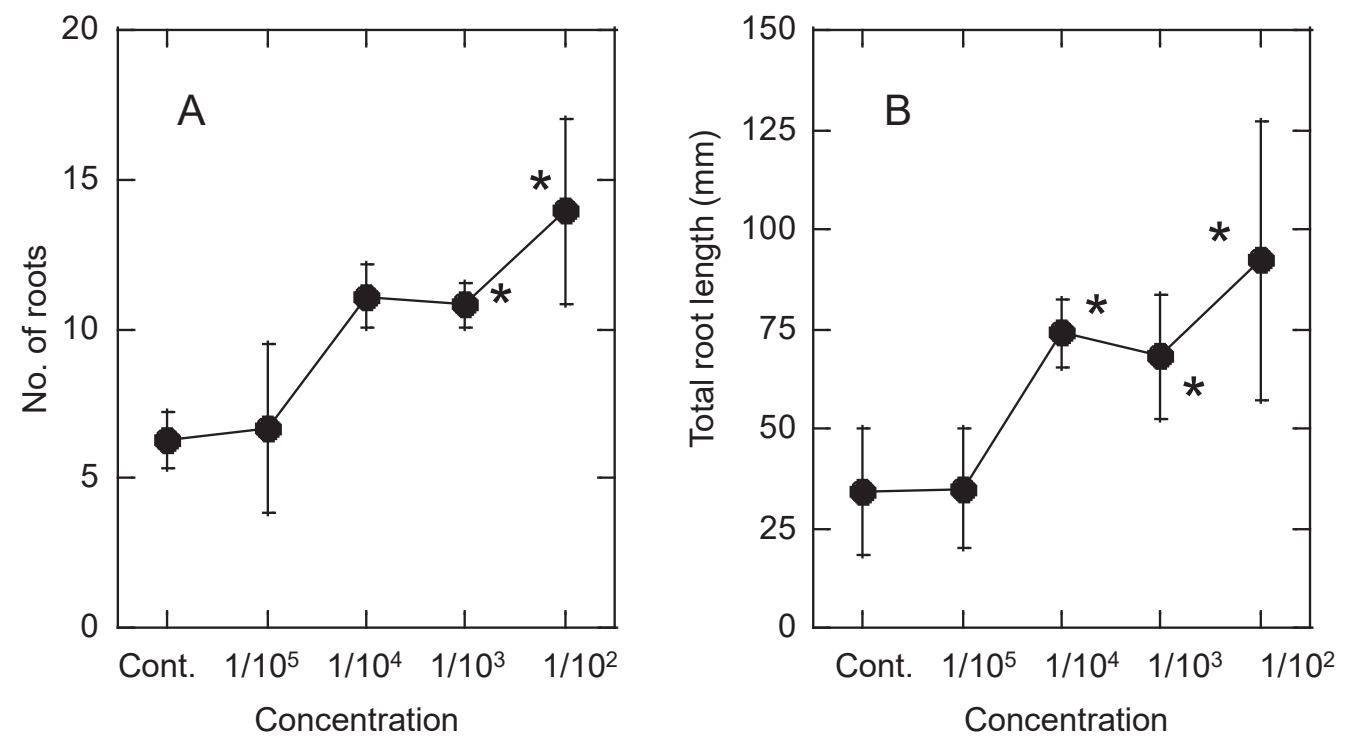

Fig. 2. Effect of NSC-Base ${ }^{\circledR}$ at different concentrations on root number (A) and total root length (B) per mung bean seedling. The concentrations of NSC-Base ${ }^{\circledR}$ are shown by the magnitude of dilution of the commercial NSC-Base ${ }^{\circledR}$ product. Data are the mean \pm SE of four experiments, each with five replicated seedlings. * shows significant difference from the control by Dunnett's multiple range test $(P<0.05)$. 

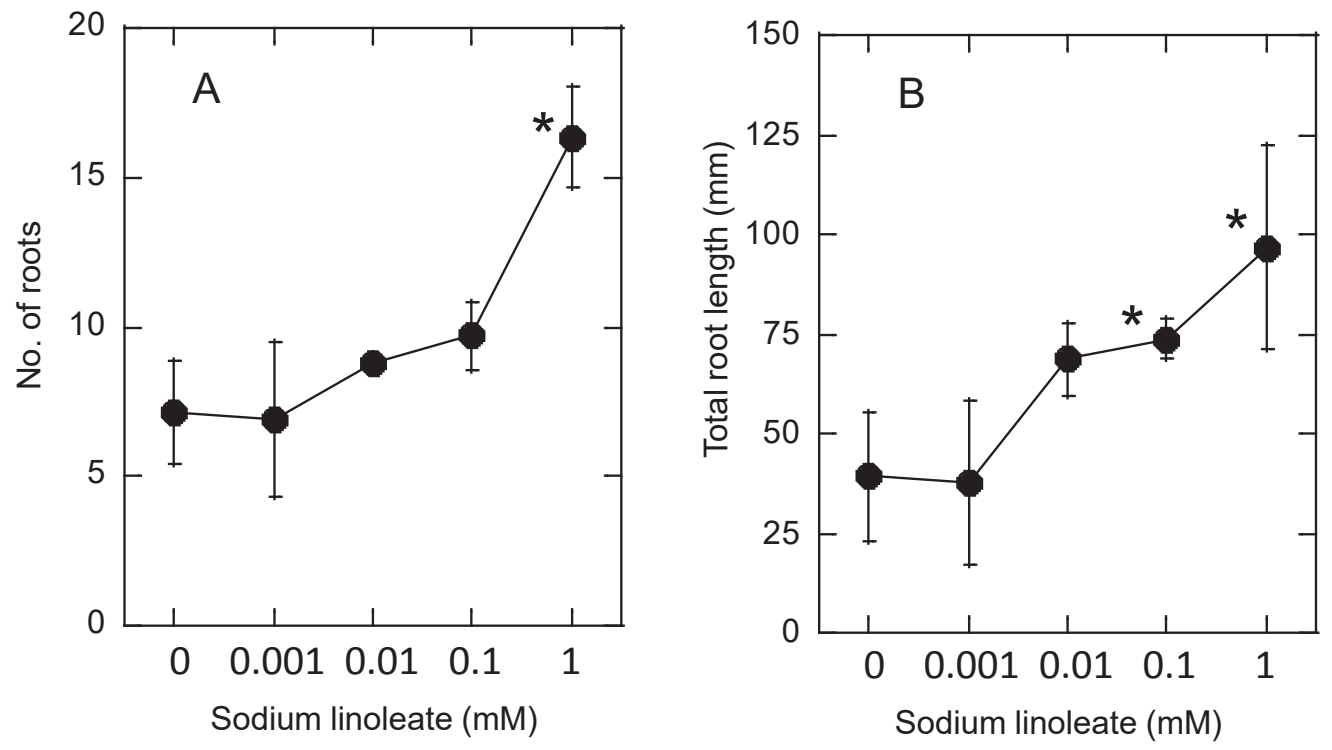

Fig. 3. Effect of sodium linoleate at different concentrations on root number (A) and total root length (B) per mung bean seedling. The concentrations were shown based on the initial concentration of added sodium linoleate as explained in Materials and Methods. Data are the mean \pm SE of four experiments, each with five replicated seedlings. * shows significant difference from the control $\left(\mathrm{H}_{2} \mathrm{O}\right)$ by Dunnett's multiple range test $(P<0.05)$.

Characterization of promotion by NSC-Base ${ }^{\circledR}$ of adventitious root formation in root-cut mung bean seedlings

In the former section, the experiments using mung bean seedlings as an experimental material were conducted with three replicated seedlings. Since some mung bean seedlings did not respond to NSCBase $^{\circledR}$ treatment and did not form adventitious roots for an unknown reason including the control and seedlings treated with NSC-Base ${ }^{\circledR}$, we selected plants typical to the treatments (Fig. 1). This feature of experimental material caused a large deviation in a single measurement in quantitative analyses, in which the number of formed roots and the total length of the root were measured. Therefore, in the following quantitative analyses, we compared the means obtained from four repeated experiments, each with five replicated seedlings.

Figure 2 shows the effects of different concentrations of NSC-Base ${ }^{\circledR}$, diluted $10^{2}$ to $10^{5}$ times, on the number of adventitious roots and the total length of the roots on each root-cut mung bean seedling. NSC-Base ${ }^{\circledR}$ diluted $10^{3}$ and $10^{2}$ times significantly increased root number and that diluted $10^{4}$ times tended to increase it. However, NSCBase $^{\circledR}$ diluted $10^{4}$ to $10^{2}$ times significantly increased total root length. NSC-Base ${ }^{\mathbb{B}}$ diluted $10^{5}$ times promoted neither root number nor total root length.

NSC-Base ${ }^{\circledR}$ is an alkaline hydrolysis product of soy oil, which consists of triglycerides containing linoleic acid (50\%), oleic acid (20\%), palmitic acid $(10 \%)$, linolenic acid $(10 \%)$ and stearic acid $(5 \%)$ as main fatty acids (Ministry of Education, Culture, Sports, Science and Technology-Japan 2015). We anticipated that sodium salts of these fatty acids might promote adventitious root formation. Then, we tested the action of these fatty acids using sodium linoleate as a candidate for possible active compounds, since its content is the largest among fatty acids contained in soy oil. The exact concentration of fatty acids in NSC-Base ${ }^{\circledR}$ has not been disclosed officially by a manufacturer, but the concentration of around $4 \%$ was obtained personally from a vender. From this value the concentration of sodium linoleate in NSC-Base ${ }^{\circledR}$ could be estimated to be $67 \mathrm{mM}$. Since NSC-Base ${ }^{\circledR}$ was active after $10^{2}$ to $10^{4}$ dilution (Fig. 2), we tested sodium linoleate at the concentration from 0.01 to $1 \mathrm{mM}$. Figure 3 shows the effects of sodium linoleate on the number of adventitious roots and the total length of the roots on each root-cut mung bean seedlings. The promotive effect of sodium linoleate increased as its concentration increased. Sodium linoleate only at 1 $\mathrm{mM}$ increased significantly the number of adventitious roots, and that at 0.01 and $0.1 \mathrm{mM}$ tended to increase it. Sodium linoleate at 0.1 and 1 $\mathrm{mM}$ increased significantly the total root length, although the chemical at $0.01 \mathrm{mM}$ seemed to increase it. 


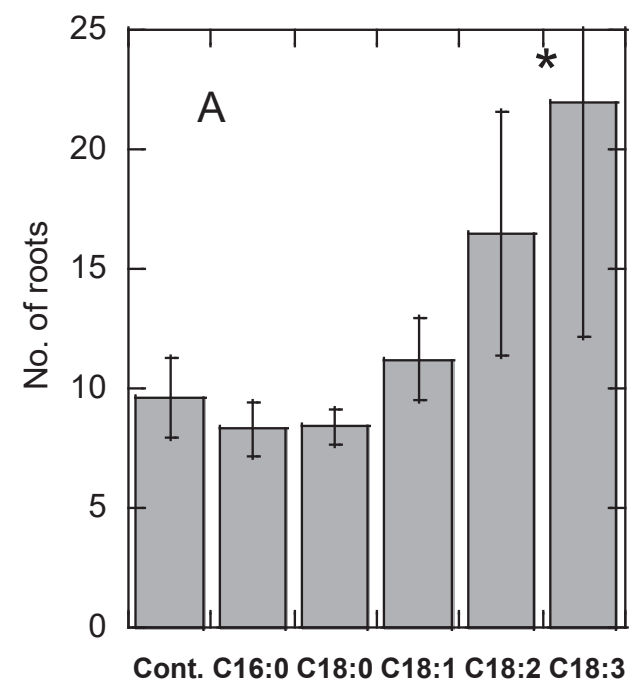

Sodium salts of fatty acids

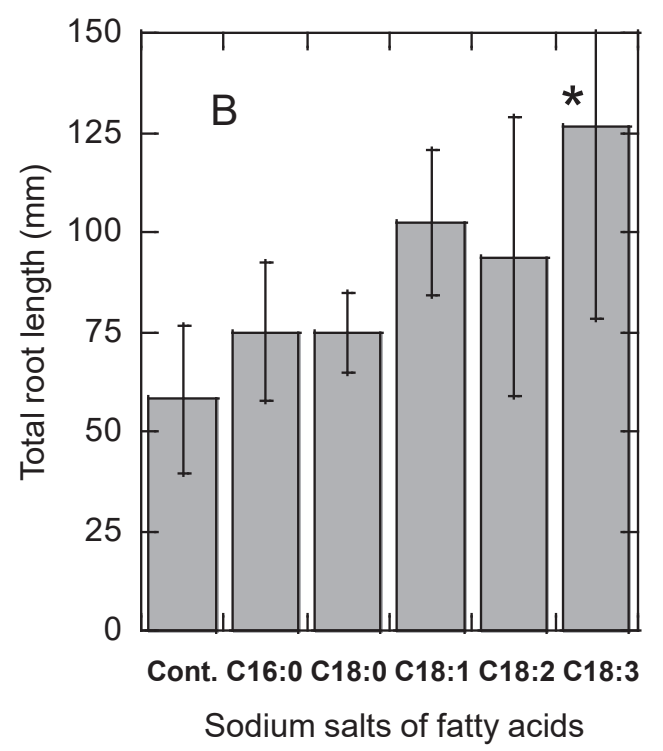

Fig. 4. Effects of respective fatty acids (sodium salts), which are included in NSC-Base ${ }^{\circledR}$, on root number (A) and total root length (B) per mung bean seedling. The concentration of fatty acid sodium salts was $1 \mathrm{mM}$, which was based on the initial concentration of added fatty acid sodium salts as explained in Materials and Methods. Data are the mean \pm SE of four experiments, each with five replicated seedlings. * shows significant difference from the control $\left(\mathrm{H}_{2} \mathrm{O}\right)$ by Dunnett's multiple range test $(P<0.05)$. C16:0, sodium palmitate; C18:0, sodium stearate; C18:1, sodium oleate; C18:1, sodium linoleate; $\mathrm{C} 18: 3$, sodium linolenate.
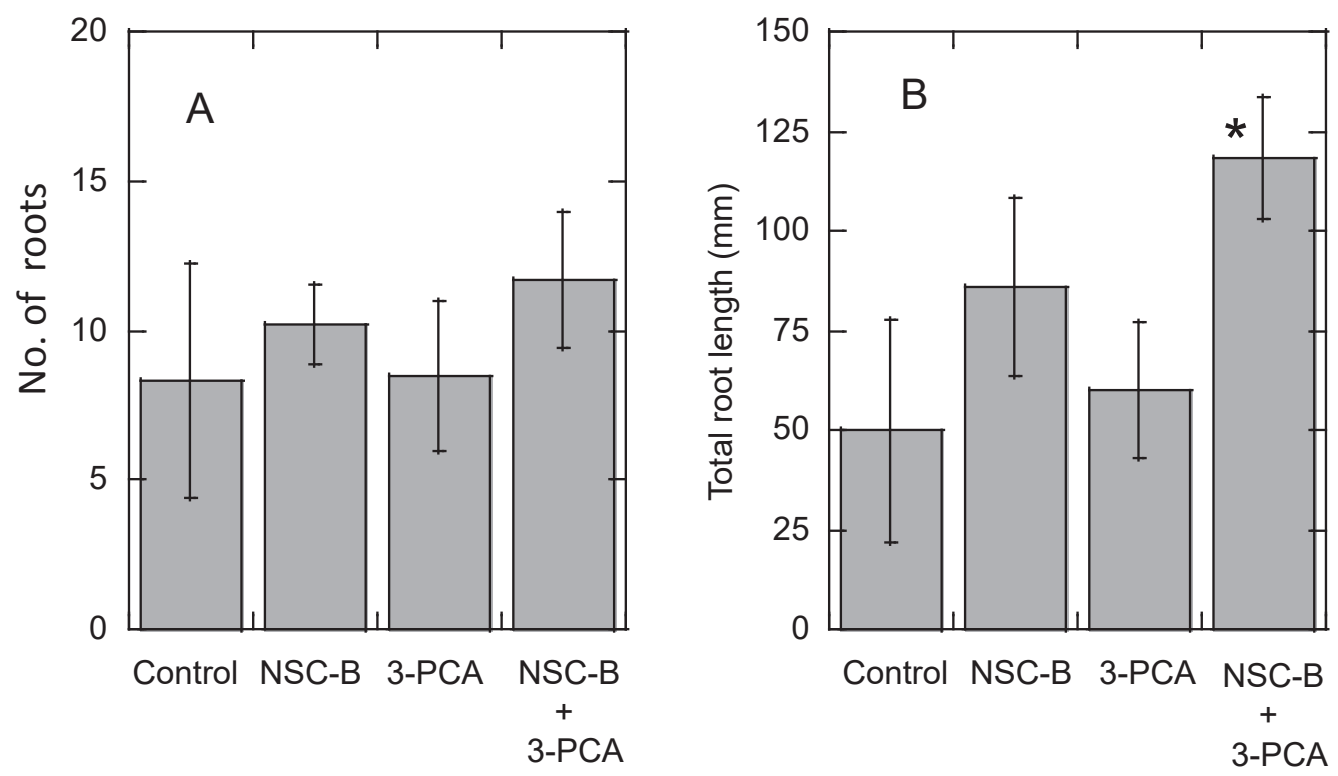

Fig. 5. Synergistic effect of NSC-Base ${ }^{\circledR}$ and 3-PCA on root number (A) and total root length (B) per mung bean seedling. NSCBase $^{\circledR}$ diluted $3 \times 10^{3}$ times and $0.3 \mathrm{mM} 3$-PCA were applied separately or in combination. Data are the mean $\pm \mathrm{SE}$ of four experiments, each with five replicated seedlings. * shows significant difference from the control by Dunnett's multiple range test $(P<0.05)$. 
Next, we examined the effect of each fatty acid in NSC-Base ${ }^{\circledR}$. Figure 4 shows that the sodium salts of oleic acid, linoleic acid and linolenic acid tended to increase the number of roots and total root length, whereas only sodium linolenate significantly increased both. Sodium salts of palmitic acid and stearic acid caused little if any increase in root number and root length. These results, combined with those shown in Figure 3B, suggested that sodium salts of unsaturated fatty acids in NSCBase $^{\circledR}$, sodium linoleate and sodium linolenate and probably sodium oleate, acted to promote adventitious root formation in root-cut mung bean seedlings, whereas saturated fatty acids, sodium palmitate and sodium stearate, did not.

Previously, Satoh and Nomura (2017) reported that 3-pyridinecarboxylic acid (3-PCA) promoted root elongation in rice seedlings. We investigated the effects of simultaneous application of 3-PCA and NSC-Base ${ }^{\circledR}$ to root-cut mung bean seedlings. Figure 5 shows that treatment with NSC-Base ${ }^{\mathbb{B}}$ diluted $3 \times 10^{3}$ times tended to stimulate adventitious root formation, but $3-\mathrm{PCA}$ at $0.3 \mathrm{mM}$ did not. Interestingly, simultaneous application of NSCBase $^{\circledR}$ and 3-PCA increased significantly total root length, suggesting a synergistic effect of both compounds. There was no difference in the root numbers among seedlings treated with or without NSC-Base ${ }^{\circledR}$ or 3-PCA alone or in combination. Collectively, these results suggest that NSC-Base ${ }^{\mathbb{B}}$ promotes adventitious root formation first, then 3-PCA stimulates elongation of the resultant roots, causing enhanced elongation of roots in root-cut mung bean seedlings.

The present results showed that sodium salts of unsaturated fatty acids contained in NSC-Base ${ }^{\circledR}$ are active in the promotion of adventitious root formation in root-cut mung bean seedlings, but saturated fatty acids in the NSC-Base ${ }^{\circledR}$ are not. We can speculate that the unsaturated acids might be oxidized to form oxylypins, epoxy compounds of long chain unsaturated fatty acids, to exert their activity to stimulate adventitious root formation. Oxylypin compounds are known to have biochemical activities in some biological systems, especially, in animals (Bach et al. 2008). The information about their involvement in plant growth regulation is scanty, excepting a relatively well analyzed 9,10- $\alpha$-ketol linolenic acid (KODA, 9-hydroxy-10-oxo-12(Z), 15(Z)-octadecadienoic acid) (Yokoyama et al. 2000). About 20 years ago, KODA was reported to have flower-inducing activity in Lemna (Yokoyama et al. 2000), and to promote rooting in cherry (Prunus 'Somei-Yoshino') cuttings (Yokoyama and Nakamura 2007) and in Swertia japonica calli (Kawakami et al. 2015).
KODA is synthesized from $\alpha$-linolenic acid by the action of 9-lipoxygenase (Yokoyama et al. 2000). The present study showed that adventitious root formation was stimulated by sodium salts of unsaturated fatty acids, probably oleic acid, linoleic acid and linolenic acid (Figs. 3 and 4), suggesting an engagement of an unknown metabolite of an oxylipin compound other than KODA, for instance vernolic acid from linoleic acid (Bafor et al. 1993, Lee 1998, Liu et al. 1998). The action mechanism of these unsaturated fatty acids, i.e., oleic acid and linolenic acid remains to be elucidated in the future.

KODA is reportedly an unstable compound itself, making difficult its direct use as a plant growth regulator (Shimomura et al. 2013). On the other hand, NSC-Base ${ }^{\circledR}$ containing a mixture of unsaturated fatty acids is currently available as a commercial specimen, and is thought to be stable in cultivation environment, making possible their use for the chemical control of plants.

\section{Acknowledgments}

We thank WIT Co., Ltd, Osaka, Japan for the kind gift of NSC-Base ${ }^{\circledR}$ and NSC-Pro ${ }^{\circledR}$. This study was supported by a Grant-in-Aid (16K07604 to S. Satoh) for Scientific Research from the Japan Society for the Promotion of Science.

\section{References}

Arteca RN 1996 Chemistry, biological effects, and mechanism of action of plant growth substances. In: Arteca RN, Plant growth substances. Chapman \& Hall, New York, USA, pp. 45-103.

Bach L, Michaelson LV, Haslam R, Bellec Y, Gissot L, Marion J, Da Costa M, Boutin J-P, Miquel M, Tellier F, Domergue F, Markham JE, Beaudoin F, Napier JA, Faure J-D 2008 The very-long-chain hydroxy fatty acylCoA dehydratase PASTICCINO2 is essential and limiting for plant development. Proc. Natl. Acad. Sci. USA 105: 14727-14731.

Bafor M, Smith MA, Jonsson L, Stobart K, Stymne S 1993 Biosynthesis of vernoleate (cis-12-epoxyoctadeca-cis-9enoate) in microsomal preparations from developing endosperm of Euphorbia lagascae. Arch. Biochem. Biophys. 303: 145-151.

Bernasconi P 1998 Auxins. In: Taiz L, Zeiger E, Eds., Plant physiology, 2nd ed. Sinauer associates, Inc. Sunderland, Massachusetts, pp. 543-590.

Hopkins WG 1995 The role of hormones in plant development. In: Hopkins WG, Introduction to plant physiology. 2nd ed., John Wiley \& Sons, Inc. New York, USA, pp. 309-334.

Kawakami H, Yokoyama M, Takagi K, Ogawa S, Hara K, Komine M, Yamamoto Y 2015 9-Hydroxy-10-oxo12(Z),15(Z)-octadecadienoic acid (KODA) enhances 
adventitious root redifferentiation from Swertia japonica callus. In Vitro Cell. Dev. Biol.-Plant 51: 201-204.

Knee EM, Hangarter RP 1996 Differential IAA dose response relations of the axr 1 and axr 2 mutants of Arabidopsis. Physiol. Plant. 98: 320-324.

Lee M, Lenman M, Banas A, Bafor M, Singh S, Schweizer M, Nilsson R, Liljenberg C, Dahlqvist A, Gummeson P-O, Sjödahl S, Green A, Stymne S 1998 Identification of non-heme diiron proteins that catalyze triple bond and epoxy group formation. Science 280: 915-918.

Liu L, Hammond EG, Nikolau BJ 1998 In vivo studies of the biosynthesis of vernolic acid in the seed of Vernonia galamensis. Lipids 33: 1217-1221.

Ministry of Education, Culture, Sports, Science and Technology-Japan 2015 Fatty acids. Table 2 . Fatty acids per $100 \mathrm{~g}$ total fatty acids. In: Standard tables of food composition in Japan -2015- (Seventh Revised Edition)

Satoh S, Nomura Y 2017. Promotion of root elongation by pyridinecarboxylic acids known as novel cut flower care agents. Plant Root 11: 40-47.

Shimomura S, Oyama S, Nakano K, Hasegawa M, Toshima H 2013 Stereoselective synthesis of a promising flower- inducing KODA analog, $(9 R, 12 S, 13 R, 15 Z)-9$-Hydroxy12,13-methylene-10-oxooctadec-15-enoic acid. Biosci. Biotech. Biochem. 77: 1354-1357.

Srivastava LM 2001 Apical dominance and some other phenomena illustrating correlative effects of hormones. In: Srivastava LM, Plant growth and development. Academic Press, San Diego, USA, pp. 303-339.

Yokoyama M, Nakamura K 2007 Upgrading of forest cultivation techniques with fast growing trees. In: International workshop on GHG sink/source control technology through conservation efficient management of terrestrial ecosystem.

Yokoyama M, Yamaguchi S, Inomata S, Komatsu K, Yoshida S, Iida T, Yokokawa Y, Yamaguchi M, Kaihara S, Takimoto A 2000 Stress-induced factor involved in flower formation of Lemna is an $\alpha$-ketol derivative of linolenic acid. Plant Cell Physiol. 41: 110-113.

Wightman F, Schneider EA, Thimann KV 1980 Hormonal factors controlling the initiation and development of lateral roots. II. Effects of exogenous growth factors on lateral root formation in pea roots. Physiol. Plant. 49: 304-314. 